\title{
Willkommen in Basel: chronische Erkrankungen - eine globale Herausforderung
}

Nino Künzlia,

Nicole Probst-Hensch ${ }^{b}$

a Prof. Dr. med. et PhD Ordinarius für Sozial- und Präventivmedizin der Universität Basel und Vizedirektor des Schweizerischen Tropenund Public Health Instituts (Swiss TPH), Leiter des Forschungsdepartements Epidemiologie und Public Health

b Prof. Dr. phil. et PhD Extraordinaria für Public Health der Universität Basel und Leiterin der Abteilung der Epidemiologie chronischer Krankheiten sowie Vize-Leiterin des Departements Epidemologie und Public Health am Swiss TPH
Korrespondenz:

Prof. Dr. med. Nino Künzli Swiss TPH

Socinstrasse 57

Postfach

CH-4052 Basel

nino.kuenzli@unibas.ch
Ende August 2011 steht Basel im Zeichen chronischer Krankheiten. Dem Workshop zum international hochaktuellen Thema von «Mega-Kohortenstudien» (24.8.2011) folgt die Jahreskonferenz von Public Health Schweiz (25./26.8.2011) zum Titelthema mit der öffentlichen Podiumsdiskussion zur Frage «Gesunde Stadt - ein Widerspruch?»

Im September folgt in New York die U.N.Gipfelkonferenz zu nichtübertragbaren Krankheiten [1]. Ein Hauptresultat soll die Verabschiedung einer Zielvereinbarung sein, um die Todesraten wegen chronischer Krankheiten jährlich um $2 \%$ zu senken. Nichtübertragbare Krankheiten sind heute weltweit die wichtigsten Todesursachen. Organisationen wie WHO oder Weltbank warnen vor den gesundheitlichen und wirtschaftlichen Folgen der globalen Epidemie chronischer Erkrankungen.

Einige Beispiele: In der Schweiz leben mindestens 4\% der Bevölkerung mit Diabetes mellitus - Dunkelziffer unbekannt. In afrikanischen Regionen südlich der Sahara wird - bei noch schlechterer Datenlage davon ausgegangen, dass in einigen Gegenden bis zu $16 \%$ an Diabetes leiden. Gemäss Hochrechnungen für Indien treten pro 1000 Einwohner jährlich ca. 2 neue Diabetesfälle auf. In der Schweiz ist bei den 45-64-Jährigen Krebs die häufigste Todesursache. Das Nationale Krebsprogramm 2011-2015 betont, dass die Krebsinzidenzrate weiterhin steigt. Erstmals liegen für die Schweiz auch Zahlen zur Inzidenz der chronisch obstruktiven Lungenerkrankung vor: gemäss SAPALDIA-Studie sind dies pro 1000 Einwohner jährlich ca. 14 neue Fälle. Die Zunahme der Lebenserwartung erklärt die Epidemie chronischer Krankheiten nur teilweise. So besteht ein grosses Potential für die Prävention.

Wichtige Ursachen dieser Krankheitslast liegen in den Folgeerscheinungen der Verstädterung unserer Lebensräume. Mehr als die Hälfte der Weltbevölkerung lebt heute in urbanem Umfeld. Damit verbunden sind starke Veränderungen des Lebensstils, der Umwelt sowie des sozialen und ökonomischen Um-

Die Swiss Public Health Conference 2011 wird von Public Health Schweiz in Zusammenarbeit mit dem Schweizerischen Tropen- und Public Health Institut organisiert. Als grösste akademische Public Health Institution der Schweiz setzt sich dieses in Forschung, Dienstleistung und Lehre für die Gesundheit der Bevölkerung ein. Public Health Schweiz setzt sich als nationale Dachorganisation für das Wachstum und die Entwicklung von Public Health und deren Umsetzung in die Praxis ein. Informationen zu den Veranstaltungen: www.public-health.ch; www. swisstph.ch

felds. Schadstoffe aus Verkehr, Industrie und Haushalt belasten Luft, Wasser und Böden. Auch in Ländern des Südens und Ostens tragen die Einflüsse von Fernsehen, Werbung und Marketing wesentlich zum gesellschaftlichen Wandel bei. Dort ersetzen chronische Krankheiten nicht die weitverbreiteten übertragbaren Krankheiten. Vielmehr tritt diese hohe Krankheitslast zusätzlich zu bestehenden Problemen. Dies stellt die Gesundheitssysteme dieser Länder vor immense Herausforderungen.

Sowohl im Süden wie im Norden bildet Forschung die zentrale Grundlage, um evidenzbasierte Strategien zur Prävention und Behandlung chronischer Krankheiten wirksam umzusetzen. Grossangelegte Langzeitstudien bilden das Rückgrat der Erforschung chronischer Krankheiten, ihrer Verläufe, Ursachen und Prävention. Das Schweizerische Tropen- und Public-Health Institut organisiert deshalb einen ganztägigen Workshop zur Zukunft der Kohortenforschung. Experten aus dem In- und Ausland werden darlegen, wie Kohorten mit mehreren Tausend Teilnehmenden aufgebaut und über Jahre erhalten werden. In geschlossenen Workshops werden dann Ziele, Inhalte, Struktur und Finanzierung der Langzeitforschung in der Schweiz diskutiert.

An zwei Tagen widmet sich die Public Health Jahreskonferenz in Symposien, Workshops, Präsentationen und Postersitzungen insbesondere den chronischen, nichtübertragbaren Erkrankungen, ihren Ursachen, ihrer Überwachung und Prävention. Die Bedeutung von Lebensstil, Umwelt und Gesundheitssystemen soll ebenso beleuchtet werden wie Chancen und Herausforderungen neuster -omics-Technologien. In der abschliessenden öffentlichen Podiumsdiskussion (26.8.2011, 15.00 Uhr) stehen Fragen zur gesunden Stadt im Zentrum. Werden unsere Städte gesundheitsgerecht geplant? Muss verdichtetes Wohnen verdichteten Lärm, verschmutzte Luft und Unfälle nach sich ziehen? Brauchen wir die «essbare Stadt»? Wie sieht die «bewegende» Stadt aus? Persönlichkeiten aus Politik, Behörden, Wissenschaft und Wirtschaft werden sich unter der Moderation von Urs Steiger, Wissenschaftsjournalist und Experte für Raumplanung und Umwelt, einer kritischen Diskussion stellen. Die Podiumsdiskussion kann ohne Anmeldung kostenlos besucht werden. Willkommen in Basel!

1 Beaglehole R et al. for The Lancet NCD Action Group and the NCD Alliance. Priority actions for the noncommunicable disease crisis. Lancet. 2011;377: $1438-47$. 